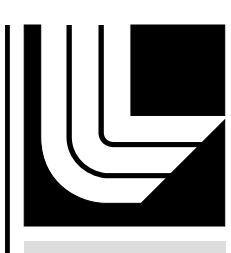

LAWRENCE LIVERM ORE NATIONAL LABORATORY

\title{
Large Scale Nanolaminate Deformable Mirror
}

A. Papavasiliou, S. Olivier, T. Barbee, R. Miles, K. Chang

December 1, 2005

SPIE MOEMS-MEMS 2006

San Jose, CA, United States

January 21, 2006 through January 27, 2006 
This document was prepared as an account of work sponsored by an agency of the United States Government. Neither the United States Government nor the University of California nor any of their employees, makes any warranty, express or implied, or assumes any legal liability or responsibility for the accuracy, completeness, or usefulness of any information, apparatus, product, or process disclosed, or represents that its use would not infringe privately owned rights. Reference herein to any specific commercial product, process, or service by trade name, trademark, manufacturer, or otherwise, does not necessarily constitute or imply its endorsement, recommendation, or favoring by the United States Government or the University of California. The views and opinions of authors expressed herein do not necessarily state or reflect those of the United States Government or the University of California, and shall not be used for advertising or product endorsement purposes. 


\title{
Large Scale Nanolaminate Deformable Mirror
}

\author{
Alexandros Papavasiliou ${ }^{\mathrm{a}}$, Scot Olivier ${ }^{\mathrm{a}}$, Troy Barbee ${ }^{\mathrm{a}}$, Robin Miles $^{\mathrm{a}}$, Kevin Chang $^{\mathrm{b}}$ \\ ${ }^{a}$ Lawrence Livermore National Laboratory, 7000 East Ave. Livermore CA 94550 \\ ${ }^{\mathrm{b}}$ MicroAssembly Technologies, 3065 Richmond Parkway Suite 109, Richmond CA 94806
}

\begin{abstract}
This work concerns the development of a technology that uses Nanolaminate foils to form light-weight, deformable mirrors that are scalable over a wide range of mirror sizes. While MEMS-based deformable mirrors and spatial light modulators have considerably reduced the cost and increased the capabilities of adaptive optic systems, there has not been a way to utilize the advantages of lithography and batch-fabrication to produce large-scale deformable mirrors.

This technology is made scalable by using fabrication techniques and lithography that are not limited to the sizes of conventional MEMS devices.

Like many MEMS devices, these mirrors use parallel plate electrostatic actuators. This technology replicates that functionality by suspending a horizontal piece of nanolaminate foil over an electrode by electroplated nickel posts. This actuator is attached, with another post, to another nanolaminate foil that acts as the mirror surface.

Most MEMS devices are produced with integrated circuit lithography techniques that are capable of very small line widths, but are not scalable to large sizes. This technology is very tolerant of lithography errors and can use coarser, printed circuit board lithography techniques that can be scaled to very large sizes.

These mirrors use small, lithographically defined actuators and thin nanolaminate foils allowing them to produce deformations over a large area while minimizing weight.

This paper will describe a staged program to develop this technology. First-principles models were developed to determine design parameters. Three stages of fabrication will be described starting with a $3 \times 3$ device using conventional metal foils and epoxy to a 10-across all-metal device with nanolaminate mirror surfaces.
\end{abstract}

Keywords: Deformable mirrors, Nanolaminates, Adaptive Optics

\section{INTRODUCTION}

Lightweight deformable mirror are needed for use in large space optics. Space telescopes with lightweight optics require adaptive elements to correct for mirror aberrations as well as atmospheric aberrations. The goal of this project is to demonstrate a technology which can be used to produce deformable mirrors with diameters on the order of 1 meter, on the order of 10,000 actuators and with an areal density on the order of $1 \mathrm{~kg} / \mathrm{m}^{2}$. We have developed a technology that combines nanolaminate foils with electrostatic actuators to form scalable, lightweight, deformable mirrors with MEMSlike actuator density.

\subsection{Previous MEMS Deformable mirrors}

Microfabrication has allowed the production of small deformable mirrors with very dense actuation. While these devices provide sufficient stroke, and high actuator density, they none of them are capable of being scaled beyond the size of a silicon wafer ${ }^{1,2}$ and many of them are limited to a single chip ${ }^{3}$.

\subsection{Nanolaminates}

Nanolaminates are thin, flexible, optically smooth metal foils with very high strength. Nanolaminate mirrors have been developed to as large light-weight passive optical elements ${ }^{4}$. However, their mechanical properties make them a good match for the high density but small force provided by electrostatic actuators. The high strength of nanolaminates makes it possible to produce a foil with a high quality optical surface that is simultaneously robust enough to survive fabrication and thin enough to be readily deformed by weak electrostatic actuators.

A nanolaminate foil is a metal foil created by sputtering thin layers of metal one on top of the other. The foils are deposited on a mandrell with a release layer that allows them to be parted from the mandrel. The with a sufficiently thin and conformal release layer, the foils take on the shape of the mandrel, and an optically smooth mandrel yields an 
optically smooth foil. The layers are deposited by magnetron sputtering over a moving table. The thickness of each individual layer as well as the whole thickness of the foil can be accurately controlled. The individual layers can be controlled to have thicknesses on the order of $1-10 \mathrm{~nm}$. The very thin layers restrict the growth of grains. The nanoscale grain size enforced by these thin layers produces a material dense with grain-boundry dislocations resulting in a very high strength. Since the material has very high strength, thin sheets of nanolaminate can be handled and will survive microfabrication. These foils can be made thin enough to allow them to be deformed by very weak electrostatic actuators but still strong enough to be handled and bonded to those actuators.

\section{DEVICE ARCHITECTURE}

The device consists of three horizontal layers separated by vertical standoffs. The base layer is a stiff layer that provides mechanical support to the whole device. The spring layer is suspended above the base layer on top of ridges. The mirror layer is attached to the spring layer with posts. When a voltage is applied between the spring layer and electrodes on the base layer, the spring layer deforms to close the gap. This deformation is translated through the posts to the mirror layer. In the prototypes described here, the base layer is made of silicon or glass, but it could be fabricated from any stiff material such as a thick nanolaminate foil. The ridges and posts described here are fabricated from SU-8 epoxy or electroplated nickel. The mirror and spring layers are fabricated of either nanolaminate foil or rolled steel and invar foils.

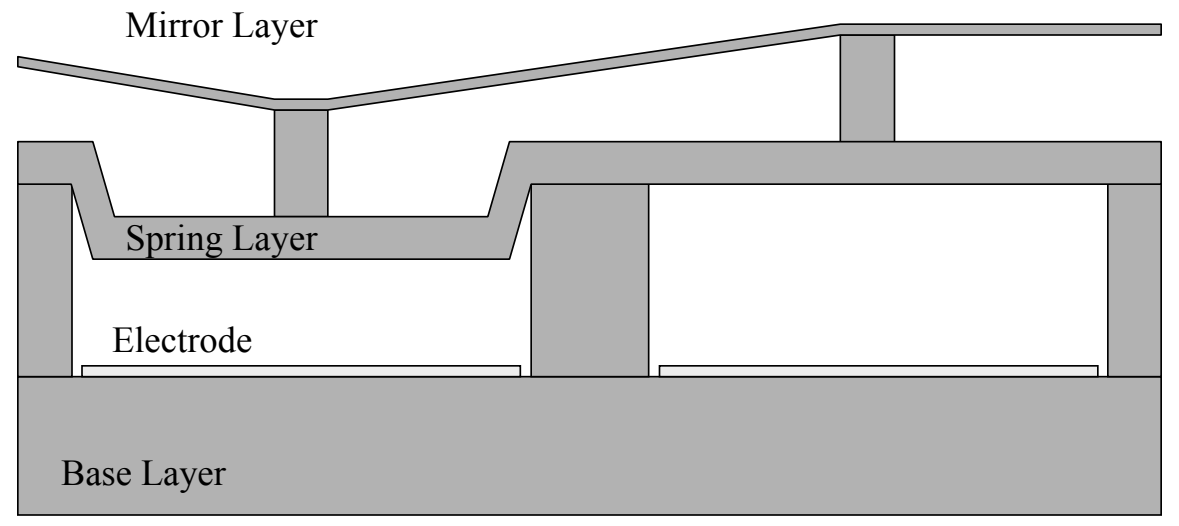

Figure 1. Cross-section of basic design.

\subsection{Actuator designs}

Two different types of spring layers have been used in this work: patterned springs, and a continuous spring layer. In the case of the patterned springs, beam shaped springs are cut into a fairly thick foil such that most of the spring layer moves in one rigid piece. In the other case, the spring layer is a thin, continuous piece of foil which deforms continuously.

The continuous design has the advantage that it does not require an additional patterning and aligning step. In addition continuous design is stable in a larger fraction of the initial gap than the patterned design. This increased stability comes as a result of the non-linearity of a deforming plate, and the fact that the portion of the plate near the edges does not move as far as the portion in the center, resulting in less overall force that the patterned actuator in which the actuator moves as a rigid plate. This disparity in overall force, however ultimately means that the patterned design requires less voltage for the same performance. 


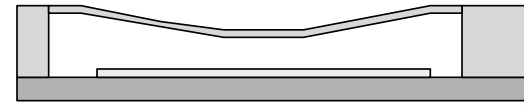

Continuous spring layer

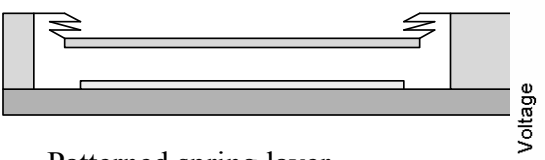

Patterned spring layer

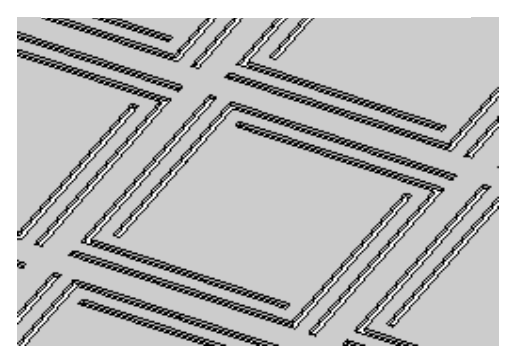

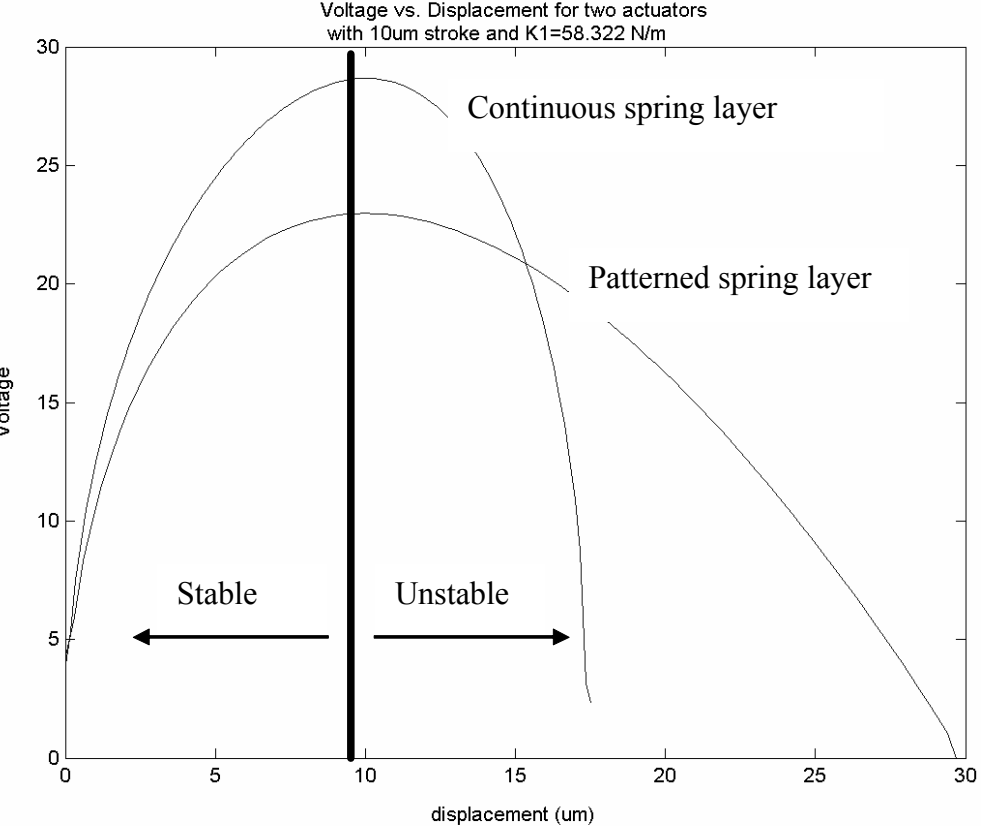

Figure 2. On the left are cross sections of the continuous and patterned spring layers as well as a drawing of the patterned spring layer design. On the right the voltage vs. displacement curve for parallel-plate with linear spring (patterned spring layer) and built-in plate (continuous spring layer) actuators. The actuators both have the same stroke $(10 \mu \mathrm{m})$ and the same spring constant. The actuators are only stable below $10 \mu \mathrm{m}$ displacement where an increase in voltage is required for an increase in displacement. The continuous spring layer only requires a $17 \mu \mathrm{m}$ gap, but requires more voltage than the patterned spring layer which requires a $30 \mu \mathrm{m}$ gap.

\subsection{Scalability}

An important goal of this project is to produce a technology that can be fabricated on the meter scale. While the architecture of the actuators is very similar to those of some parallel-plate MEMS deformable mirrors, the microfabrication technology used to produce those devices is not scalable to the sizes required here. To accomplish MEMS-like functionality, without microfabrication, we have implemented coarser lithography techniques and electroplating to produce these devices.

\subsubsection{Lithography}

Conventional microfabrication lithography is limited to the size of silicon wafers. The current state-of-the-art is $300 \mathrm{~mm}$ diameter. To produce mirrors on the order of 1 meter and larger, we designed our devices to be compatible with printed circuit board lithography. Since the pitch of the actuators in these devices is much larger than that of similar MEMS DMs, these devices are much more tolerant of lithography errors. We simulated the deviation in drive voltage as a function of deviations from the nominal actuator area. We found that a lithography error of $50 \mu \mathrm{m}$, larger than those expected with dry-film resist lithography, produced a drive voltage deviation of only $0.8 \%$

\subsubsection{Electroplating}

Like the lithography of silicon MEMS devices, the production of consistent silicon films and the patterning of those films is limited to the size of silicon wafers. In order to fabricate devices on a much larger scale, we used electroplated metal to produce the vertical elements of the DM. The metal is electroplated up through a photoresist mask, then planarized to control the thickness of the posts and ridges. The thickness of the devices produced to date have variations on the order of 2-3.5 $\mu \mathrm{m}$. This should result in $10 \%-15 \%$ drive voltage variation.

While to-date only 4" wafer sized samples have been produced, the technology exists to extend this production technique to larger scales. Very precise thickness control can be achieved by a combination of localized addition and removal of material along with localized thickness measurements. Brush plating can be used to add material locally by 
concentrating the field and plating solution in a localized area where not enough material had been deposited earlier. Small-tool grinding can be used to remove material from areas where excess material has been deposited.

\subsection{Areal Density}

One of the goals of this project is to produce a mirror technology that is lightweight with an areal density on the order of $1 \mathrm{~kg} / \mathrm{m}^{2}$. Since the nanolamiante layers are thin enough to be flexible, they are also lightweight. A representative device with $15 \mu \mathrm{m}$ thick mirror layer, $7 \mu \mathrm{m}$ thick spring layer and a $200 \mu \mathrm{m}$ thick base layer has a areal density of $2.32 \mathrm{~kg} / \mathrm{m}^{2}$.

\subsection{Bonding}

The bonding work in this project was performed by MicroAssembly Technologies. The original plan for this project was to use the metal compression bonding. However, the success of the die-attach epoxy used in early devices and the fact that it requires no additional heating made a compelling case for moving to using epoxy exclusively. Early devices showed significant print-through due to leakage of the epoxy away from the bonding location. The leakage has been minimized by reducing the volume of epoxy and increasing the accuracy of the deposition. This has resulted in greatly reduced print-through.

\section{MODELING AND DESIGN}

We modeled the deformable mirror in order to determine design parameters that will produce a working device.

\subsection{Model}

We produced a parametric model of the deformable mirror by first modeling the actuator, then the mirror, then combining the two.

\subsubsection{Actuator model}

\subsubsection{Patterned spring layer design}

The patterned spring layer was modeled as a parallel-plate actuator with linear springs. The springs were modeled as fixed-guided beams. The beams in this design are not constrained from moving laterally so no non-linear terms were added to account for elongation.

The electrostatic force in a parallel plate actuator is given by:

$$
F=\frac{1}{2} \frac{\varepsilon_{o} A V^{2}}{(g-z)^{2}}
$$

The spring constant of linear fixed-guided beams is given by:

$F=K \times Z$

The spring and electrostatic forces can be balanced with the force applied by the mirror to solve for displacement as a function of voltage.

\subsubsection{Continuous spring layer design}

The continuous spring layer model is complicated by a non-uniform force and a non-linear resistance to deformation. The electrostatic force applies a non-uniform, deformation and position dependent force on the continuous spring layer. The spring layer is built-in at the edges and as a result, the plate must elongate as well as bend. Since the peak deformations will be on the order of the thickness of the plate, the resistance to elongation must be accounted for. This actuator was modeled by assuming a deformed shape then calculating both the strain energy and the change in electrostatic energy. These energies are balanced to determine the stable deformation.

We approximated the shape of the deformed plate as a double cosine curve. This is the exact solution for small deformations with a uniform load. Although the deformation of the actual plate is likely to be different due to the concentration of electrostatic load near the center, this deviation will be small.

The deformation is given by:

$\left.Z_{(x, y)}=\frac{A}{4}\left\{1-\cos \left[\frac{2 \pi}{l}\left(x-\frac{l}{2}\right)\right]\right\} 1-\cos \left[\frac{2 \pi}{l}\left(y-\frac{l}{2}\right)\right]\right\}$ 
where $\mathrm{A}$ is the amplitude, and 1 is the lateral dimensions of the square plate.

The strain energy of the deformed plate due to bending can be written as

$d U_{\text {bending }}=\frac{1}{2} D\left\{\left(\frac{\partial^{2} z}{\partial x^{2}}+\frac{\partial^{2} z}{\partial y^{2}}\right)^{2}-2(1-v)\left[\frac{\partial^{2} z}{\partial x^{2}} \frac{\partial^{2} z}{\partial y^{2}}-\left(\frac{\partial^{2} z}{\partial x \partial y}\right)^{2}\right]\right\} d x d y$

The energy due to stretching is:

$d U_{\text {stretching }}=\frac{E t}{8\left(1-v^{2}\right)}\left[\left(\frac{\partial z}{\partial x}\right)^{4}+\left(\frac{\partial z}{\partial y}\right)^{4}+2 v\left(\frac{\partial z}{\partial x}\right)^{2}\left(\frac{\partial z}{\partial y}\right)^{2}\right] d x d y$

The electrostatic energy of stored in the gap is a function of the displacement over the area of the plate.

$U=\iint 1 / 2 \frac{\varepsilon_{o} V^{2}}{\left(g-z_{(x, y)}\right)} d x d y$

\subsection{Mirror Model}

Mirror Model The next important portion of the model is the mirror. The mirror in this case will be a continuous nanolaminate foil attached by posts to the MEMS actuators. In order to design a system that will achieve high-spatialfrequency shapes, we need to model how the mirror resists bending into those shapes. The governing equation of the deformation of the mirror can be written as:

$$
\nabla^{4} z=\frac{1}{D}\left(q+\frac{1}{2} \frac{E h}{\left(1-v^{2}\right)}\left(\left(\frac{\partial z}{\partial x}\right)^{2} \frac{\partial^{2} z}{\partial x^{2}}\right)+\left(\left(\frac{\partial z}{\partial y}\right)^{2} \frac{\partial^{2} z}{\partial y^{2}}\right)\right)
$$

Unlike the actuator plate which could be simplified to a plate with deformations that only vary with one horizontal direction, the mirror definitely deforms with respect to both $\mathrm{X}$ and $\mathrm{Y}$. Thus the solution for the problem is a partial differential equation instead of an ordinary differential equation. Numerically solving a PDE is much more challenging than an ODE. We solved this problem by simplifying it to a repeating high-spatial-frequency pattern for which a solution could be found.

\subsubsection{Checkerboard pattern}

We chose to model the mirror with a "checker board" actuation pattern where every other actuator is pulled down with the maximum potential and the alternate actuators are not actuated. The checkerboard is a repeating high-spatialfrequency shape that can be easily modeled. It can be seen from inspection that each element in a checkerboard loaded plate is a square plate with a point load in the center and simply supported at each edge.

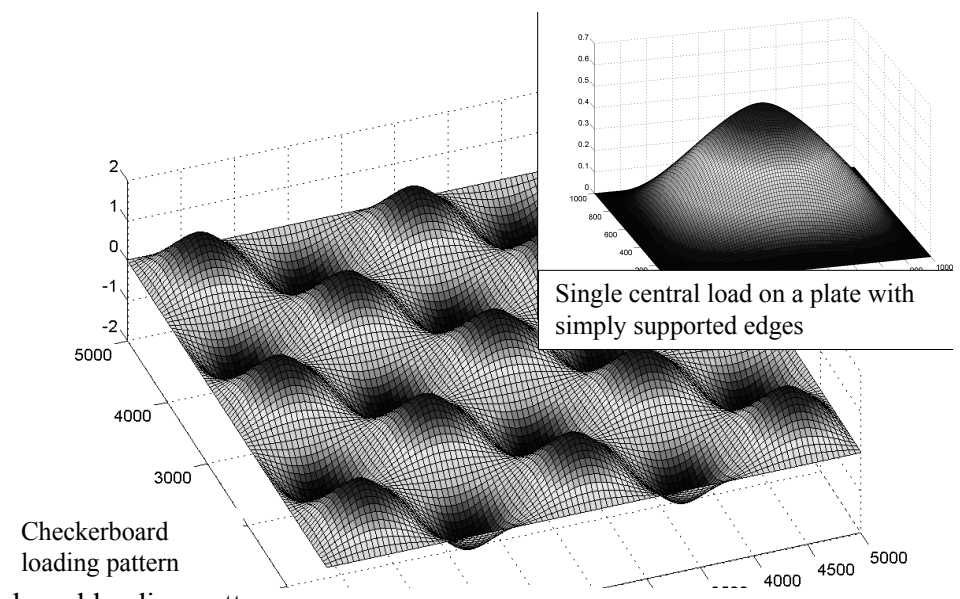

Figure3. Solution to checkerboard loading pattern 


\subsubsection{Plate Simulation}

We found the shape of the deformation by solving for the simpler case of small deflections, where the resistance of the plate to stretching is considered negligible. The small deflection approximation is a good assumption when the deflections are smaller than the thickness of the plate. In this case, the deflections are on the order of the thickness of the plate and resistance to stretching should be considered. However, it is generally accepted that a good approximation to the true resistance to deformation can be found by using the small deflection approximation to find the shape, then finding the restoring force by calculating the strain energy of the small deflection shape ${ }^{4}$. In this case, the governing equation for the small deflections is:

$\nabla^{4} z=\frac{q}{D}$

If the loads are written as the sum of a series of sine functions, the deformation will be the fourth integral of those loads, and thus a series of the same sine functions. Thus for a rectangular plate Navier's solution to this problem is to create a geometric series ${ }^{4}$ :

$z(x, y)=\frac{1}{\pi^{4} D} \sum_{m=1}^{\infty} \sum_{n=1}^{\infty} \frac{a_{m n}}{\left(\frac{m^{2}}{l_{x}^{2}}+\frac{n^{2}}{l_{y}^{2}}\right)^{2}} \sin \left(\frac{m \pi x}{l_{x}}\right) \sin \left(\frac{n \pi y}{l_{y}}\right)$

where $\mathrm{a}$ is a matrix of constants determined by the loading conditions such that

the load is

$$
q(x, y)=\sum_{m=1}^{\infty} \sum_{n=1}^{\infty} a_{m n} \sin \left(\frac{m \pi x}{l_{x}}\right) \sin \left(\frac{n \pi y}{l_{y}}\right)
$$

Note that the edges of this solution will always have a position of zero, a finite slope, zero curvature corresponding to zero moment, a finite third derivative corresponding to a finite shear, and a zero fourth derivative corresponding to zero load. So, the solution accurately describes a simply supported edge.

For a point load at the center of a rectangular plate, the matrix a can be found to be:

$a_{m n}=\frac{4 F}{l_{x} l_{y}} \sin \left(\frac{m \pi}{2}\right) \sin \left(\frac{n \pi}{2}\right)$

The deflection at any point on the plate can now easily be found as a function of plate geometry and the magnitude of a central point load $\mathrm{P}$.

To find the large-deflection restoring force, we use the large deflection equations for strain energy shown above.

From this we can find the strain energy and the restoring force as functions of displacement. We wrote a MATLAB script to calculate the matrix of coefficients for a unit point load and, from that, the shape of the deformed plate. A second MATLAB script calculated the energy as a function of the shape. We performed this calculation for plates with a variety of plate parameters and simplified the equations for energy and force to a cubic stiffening spring. The spring between two adjacent pixels is actually two plate springs in series and the resulting stiffness of the system is:

$$
K_{1}=43.19 \frac{D}{L^{2}} \text {, and } \quad K_{3}=\frac{E t}{L^{2}}\left(0.9995+0.1751 v+0.6804 v^{2}+2.588 v^{3}\right)
$$

\subsection{Integrated system}

With models for the two major components of the deformable mirror, the next problem is to combine the two to find the high-spatial-frequency response of the entire system. One of the advantages of the checkerboard pattern is that there are an equal number of actuated and un-actuated pixels. Thus the force applied by each actuated pixel on the mirror must equal the force applied by the mirror on each un-actuated pixel. 


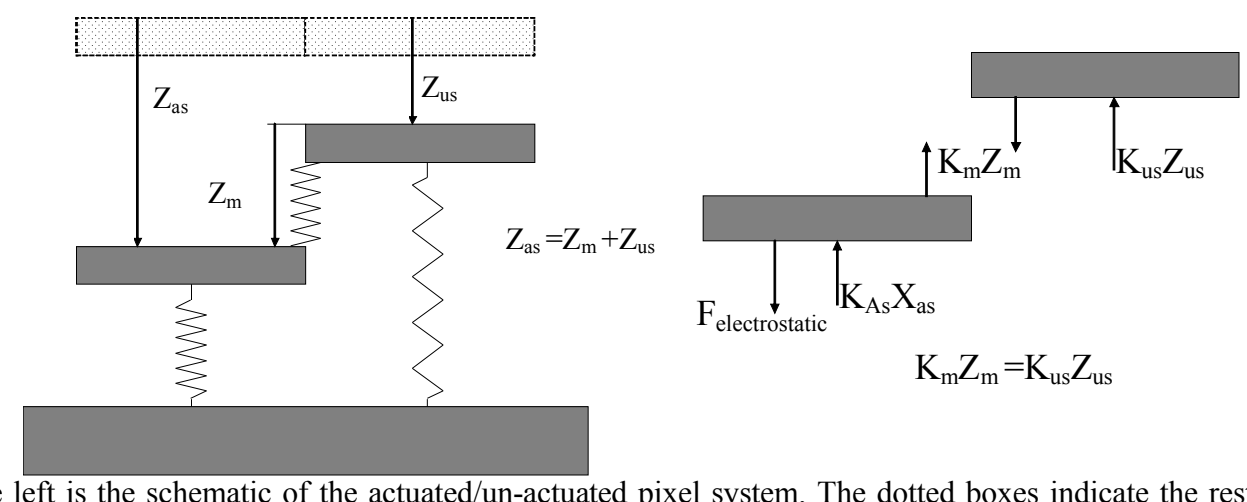

Figure4.On the left is the schematic of the actuated/un-actuated pixel system. The dotted boxes indicate the rest position of both pixels, the grey boxes indicate the deformed position of the pixels. The pixel on the left is pulled down electrostaically, the pixel on the right is pulled down by the mirror. The mirror is represented by a sprig between the two pixles. On the right is the free-body diagram of the two pixels.

As shown in the diagram above, the deflection of the actuated pixel, $Z_{\text {as }}$ must be equal to the sum of the deflection of the mirror $Z_{\mathrm{m}}$ and the un-actuated pixel $Z_{\mathrm{us}}$. From the free-body diagram of the two pixels, the force translated through the mirror must be equal to the restoring force of the deformed mirror and the restoring force of the un-actuated pixel. From the actuator model, we can find the electrostatic force as a function of displacement. This gives us 3 equations and 3 unknowns, so we can solve for the displacement of all three portions of the system.

\subsection{Design}

We tested a number of different spring layer thicknesses and found the influence and maximum voltage for those thickenesses. Note the gap increases as the thickness of the spring layer increases because the spring becomes less cubic.

Voltage as a function of influence is shown in the following chart:

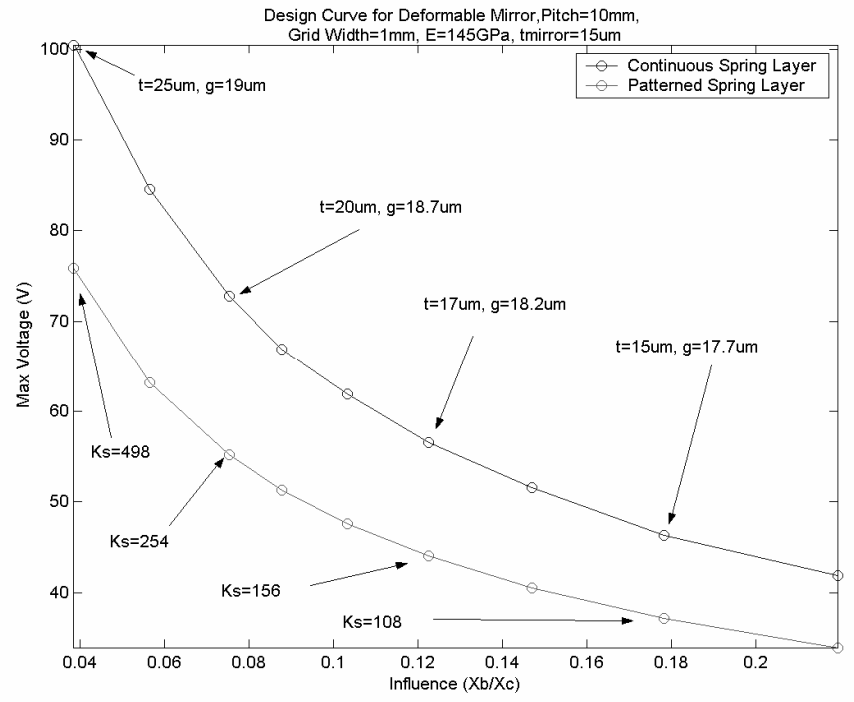

Figure 5. Design space for DM

We find that there is a fairly large space for designs that will probably work. We can make a design that uses 100 volts and achieves an influence function of 0.04 , or a device that uses as little as 50 volts but has an influence function of around 0.18 . We can make a device that has less influence or less voltage by patterning the spring layer. This large feasible design space means that if necessary, a thicker mirror or spring foil could be used to increase "handleability." 


\section{FABRICATION}

This project was executed in a phased approach starting with a small array made of convenient materials working up to a 10-across array nanolaminate deformable mirror.

Table 2. Device development

\begin{tabular}{|l|l|l|l|}
\hline & $1^{\text {st }} 3 \times 3$ & $2^{\text {nd }} 3 \times 3$ & 10 -across \\
\hline Pixel count & 9 & 9 & 76 \\
\hline Pitch & $1 \mathrm{~cm}$ & $1 \mathrm{~cm}$ & $9 \mathrm{~mm}$ \\
\hline Base material & Glass & Silicon & Silicon \\
\hline Ridge Material & SU-8 epoxy & Electroplated Nickel & $\begin{array}{l}\text { Electroplated } \\
\text { Nickel/Sputtered } \\
\text { Aluminum }\end{array}$ \\
\hline Spring layer & Rolled Invar foil & Nanolaminate/Rolled invar & $\begin{array}{l}\text { Nanolaminate/rolled } \\
\text { Stainless steel }\end{array}$ \\
\hline Post material & SU-8 epoxy & Electroplated Nickel & $\begin{array}{l}\text { Electroplated Nickel/ } \\
\text { Sputtered Aluminum }\end{array}$ \\
\hline
\end{tabular}

\subsection{First Generation $3 \times 3$}

The first generation device was a $3 \times 3$ array proof of principle device using materials that were easily obtainable and easily put together. The base layers of these devices were glass wafers. Ridges were formed on the wafers by patterning SU-8 epoxy. Rolled invar layers were used as the mirror and spring layers. Two devices were produced. One has a patterned spring layer the other has a continuous spring layer. Both of these devices were bonded with die attach adhesive.
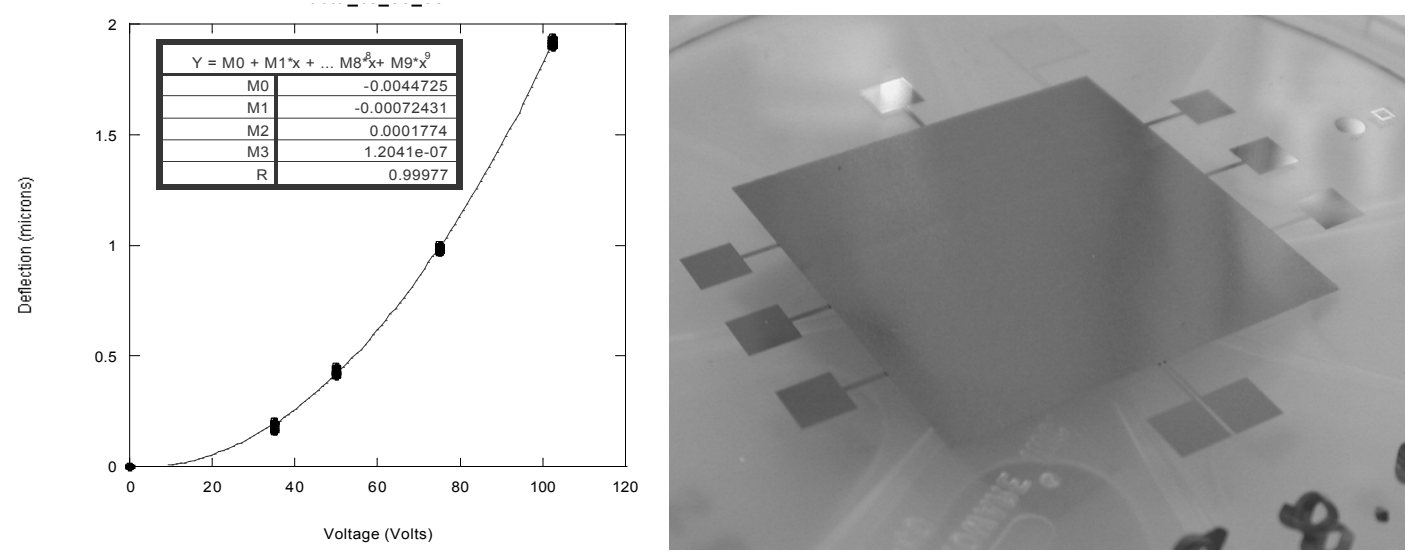

Figure 6. Deflection vs. Voltage for first generation device, photograph of first generation device.

\subsection{Second Generation $3 \times 3$}

The second generation $3 \times 3$ devices were fabricated with electroplated metal posts and ridges and with nanolaminate mirrors.

For these devices, nickel ridges were electroplated on a silicon wafer and nickel posts were fabricated on the back side of nanolaminate foils. Seven of these devices were produced with continuous nanolaminate foil spring layers. One device was produced with a patterned invar spring layer. One additional test device was produced with a nanolaminate spring layer but no mirror layer.

\subsubsection{Effect of Foil curvature}

The devices produced have displayed a significant amount of curling at the edge and a significant number of shorted pixels. We believe both of these are symptoms of the curvature of the nanolaminate foils. The nanolaminate 
development section of this report will include a section on the issues related to the flattening of nanolaminate foils, however, it is important to note here that the curling of the foils has a significant impact on the performance of the devices.

Curling in the mirror layer is caused by a residual moment in the foil. When a curled mirror layer is attached to the rest of the device, the spring layer must provide enough moment to flatten the mirror layer. However, the spring layer can only provide a very small amount of moment. The spring layer is fairly weak, designed to produce enough force to induce around $40 \mathrm{~cm}$ radius of curvature in the mirror layer. The mirror layers used for this part of the project were significantly more curly than $40 \mathrm{~cm}$ easily overwhelming the spring layer. Some of the spring pixels are bottomed out causing shorts. Others are pulled up away from their base-layer electrodes, greatly decreasing their sensitivity to applied voltage.

We believe that curling in the spring layer should have only a minimal effect on the performance of the spring layer. The spring layer is built in at all four sides of each pixel. The rigid attachment should provide sufficient moment to flatten the foil across each pixel. This theory is borne out by the fact that none of the pixels in the device with out a mirror layer were shorted.

\subsubsection{Bonding:}

Experiments with the $2^{\text {nd }}$ generation $3 \times 3$ device have allowed substantial improvement in the epoxy bonding process. Shown below are two devices, the first and the most recent device. The print-through evident in the first device is greatly reduced in the second. The print-through in the first device was caused by leakage of excess epoxy away from the bond area. The volume of epoxy used was reduced and the precision of the application was increased to minimize this leakage. The result is a drastic reduction in print-through.
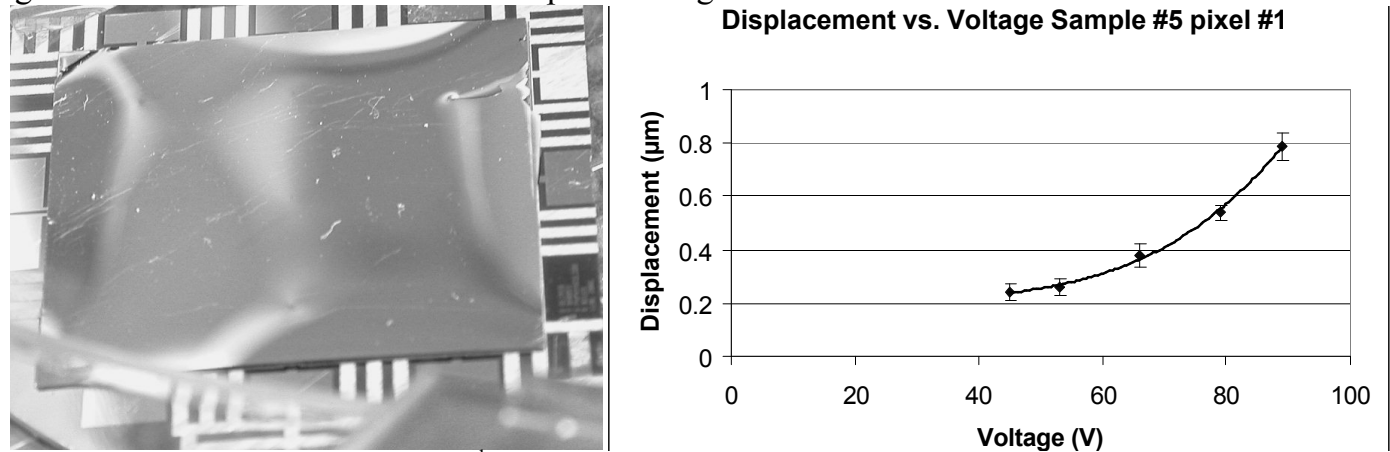

Figure 7. Deflection vs. Voltage plot for a $2^{\text {nd }}$ generation $3 \times 3$ device with a continuous nanolaminate spring layer, a nanolaminate mirror layer and electroplated nickel posts and ridges. The magnitude of deflection is less than expected. This may be due to the mirror layer pulling the spring layer away from the base layer resulting in a much larger than designed gap and an decrease in electrostatic force.

\subsection{0-across}

Parts for 10-across nanolaminate deformable mirrors have been produced and are awaiting the results from the experiments done on the $1 \mathrm{~cm} 2 \times 2$ devices. The parts delivered include: base layers with electroplated ridges, continuous nanolaminate spring layers, patterned stainless steel spring layers 6 nanolaminate mirror layers with electroplated posts. In addition there are currently base layers with sputtered aluminum ridges and mirror layers with sputtered aluminum posts in production. The mirror layers produced for the 10-across devices were produced after the work described in the nanolaminate section on reducing foil curling. As a result, the 10-across devices should have a higher likelihood of success than the $3 \times 3$ devices did.

\subsubsection{Mounting and packaging:}

The mounting and packaging scheme developed for this project consists of an optical flat glass plate onto which both the deformable mirror and a printed circuit board will be glued. Standard electrical wire bond connections will be made between the mirror and the board. Ribbon cable will connect the board to external electronics. 


\section{CONCUSION}

We have demonstrated a technology that can be scaled to the meter class and is capable of producing deformable mirrors with the required actuator density and deformations. We have fabricated small scale versions that demonstrate the potential of larger devices. We are currently fabricating a 10 -across device with over $9 \mathrm{~cm}$ of clear aperture which will further demonstrate the practicality of this fabrication technique.

\section{ACKNOWLEDGEMENTS}

This work was performed under the auspices of the U.S. Department of Energy by University of California, Lawrence Livermore National Laboratory under Contract W-7405-Eng-48.

\section{REFERENCES}

1. Mansell, J., and Byer, R.; "Silicon Micromachined Deformable Mirror." SPIE-Int. Soc. Opt. Eng. Proceedings of Spie - the International Society for Optical Engineering, vol.3353, 1998, pp.896-901. USA

2. Kruczynski, P., Bogart, B., Lai, W., Lifton, V., Mansfield, W., Tyson, J., Sadoulet, B., and Williams, D., Electrostatically actuated membrane mirrors for adaptive optics, SPIE-Int. Soc. Opt. Eng. Proceedings of Spie - the International Society for Optical Engineering, vol.4983, 2003, pp.305-313. USA

3. Bifano T, Bierden P, Perreault J. Micromachined deformable mirrors for dynamic wavefront control. SPIE-Int. Soc. Opt. Eng. Proceedings of Spie - the International Society for Optical Engineering, vol.5553, no.1, 12 Oct. 2004, pp.1-16. USA.

4. Hickey GS, Shyh-Shiuh Lih, Barbee TW Jr. Development of nanolaminate thin-shell mirrors. SPIE-Int. Soc. Opt. Eng. Proceedings of Spie - the International Society for Optical Engineering, vol.4849, 2002, pp.63-76. USA.

5. Timoshenko, S. and Woinowsky-Krieger, S.; Theory of Plates and Shells, McGraw-Hill, NewYork, 1959 